\title{
Robotic Assisted Radical Prostatectomy: Surgical Techniques and Outcomes
}

\author{
Jose R. Colombo Jr, Bruno Santos, Jason Hafron, Troy Gianduzzo, Georges-Pascal Haber, \\ Jihad H. Kaouk
}

Section of Laparoscopic and Robotic Surgery, Glickman Urological Institute, Cleveland Clinic, Cleveland, Ohio, USA

\begin{abstract}
Robotic assisted radical prostatectomy (RARP) is gaining acceptance and popularity among urologists all over the world. Early oncologic and functional results are encouraging. In this manuscript, we describe in detail both approaches for RARP and show the results of the robotic surgery program with over 300 RARP performed at our institution.
\end{abstract}

Key words: prostate cancer; laparoscopy; robotic surgery; prostatectomy

Int Braz J Urol. 2007; 33: 803-9

\section{INTRODUCTION}

Robotic assisted radical prostatectomy (RARP) provides several advantages such as precise dissection through improved instrument control with articulating tips, 3-dimensional vision and magnified view (10x), intuitive eye-hand coordination, motion scaling and filter of tremor. However, the best benefit offered by the surgical robotic is minimizing the steep learning curve set by the laparoscopic approach for radical prostatectomy (1).

Since it was first described in $2001(2,3)$ the robotic assisted radical prostatectomy (RARP) is gaining acceptance and popularity among urologists all over the world. Our minimally invasive radical prostatectomy program was initiated in 1999 with over 1000 cases performed at this time. The robotic program was introduced in 2001 after a significant experience with the pure laparoscopic approach, with over 350 robotic cases performed up to date. Herein we describe in detail, the step-by step surgical technique of RARP, using the da Vinci S Surgical System (Intuitive Surgical, Sunnyvale, California, USA), and also discuss the perioperative outcomes.

\section{SURGICAL TECHNIQUE}

\section{Transperitoneal Approach}

Patient preparation: A minimum period of 6 weeks between the prostate biopsy and the operation is recommended. Anti-platelet agents are discontinued 2 weeks prior to the surgery and a clear liquid diet is given $18 \mathrm{hrs}$ before the surgery.

Patient positioning: All patients receive prophylactic parenteral third generation cephalosporin antibiotics and compression stockings prior to the procedure. No subcutaneous heparin is used. After induction of general endotracheal anesthesia, the 


\section{Robotic Assisted Radical Prostatectomy}

patient is positioned in the supine position and is adequately padded at all pressure points, with arms placed at patient's side. The legs are separated in semi flexion (lithotomy position) and a 20F Foley catheter is inserted into the bladder. The operating table is maintained in a significant Trendelenburg position for the duration of the procedure (Figure-1).

Pneumoperitoneum: A Veress needle is inserted at the periumbilical position, the pneumoperitoneum is established initially at $20 \mathrm{mmHg}$

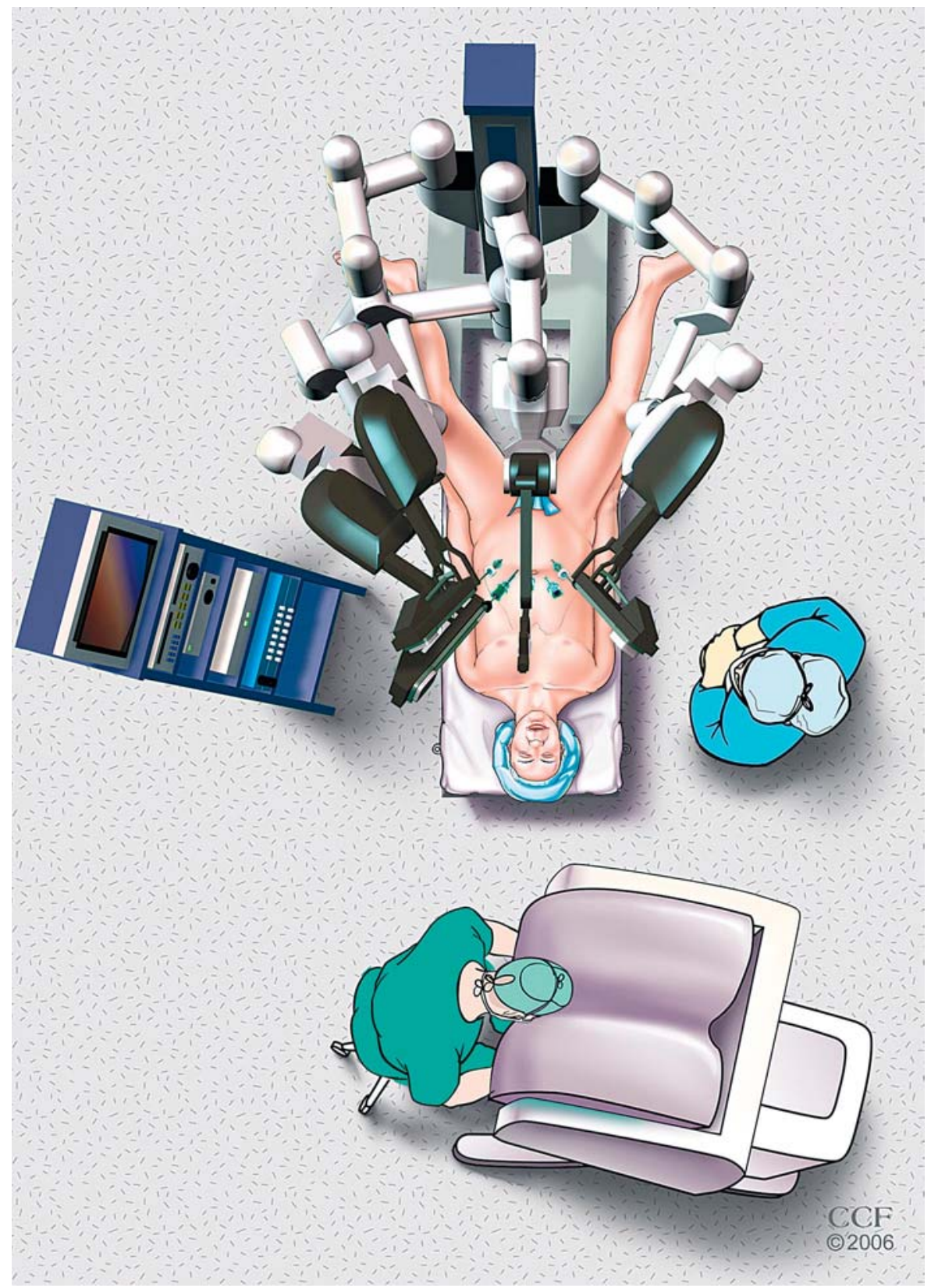

Figure 1 - Patient placed in the supine position with the legs spread to accommodate the robot. Note that the primary assistant is positioned on the right side of the patient and the 4th robotic arm used for traction is positioned at the left side at the patient's pelvis. 
for adequate port positioning, and then lowered to 12$15 \mathrm{mmHg}$.

Port placement: The Veress needle is replaced by a $12 \mathrm{~mm}$ port and the laparoscope is introduced for initial abdomen inspection. Under direct vision, four additional trocars are placed: three $8 \mathrm{~mm}$ da Vinci trocars and an extra $12 \mathrm{~mm}$ regular port are positioned as shown in Figure-2.

Bladder mobilization: After a complete inspection of the abdominal cavity performed using a $30^{\circ}$ scope, lysis of adhesions is performed. After docking the robot, the extraperitoneal space is entered through an inverted U-shaped incision in the parietal peritoneum is made, superiorly to include the urachus, and laterally to medial umbilical ligaments. The third robotic arm, available in the new da Vinci system, is used to assist in counter traction and exposure. After the medial umbilical ligaments and urachus are incised, and the Retzius space entered, the pubic bone and the iliac vessels are identified and exposed (Figure-3).

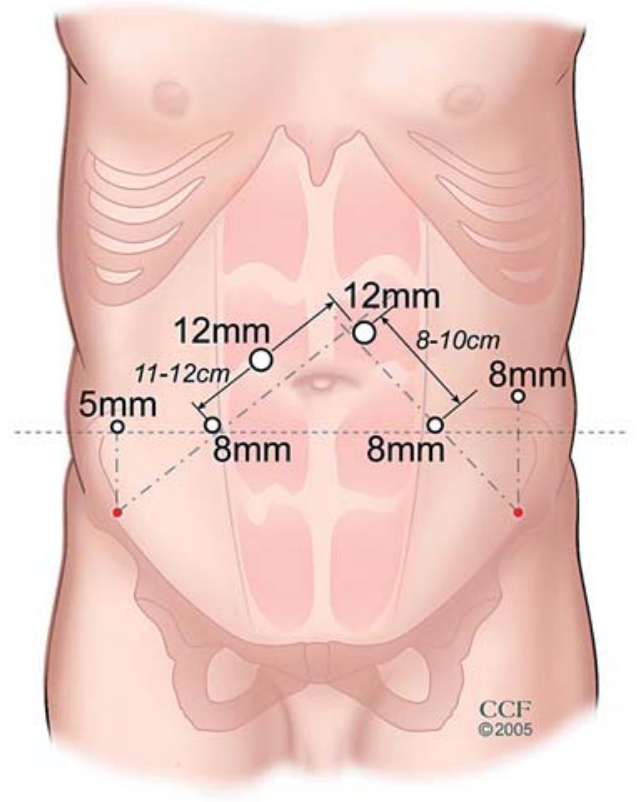

Figure 2-Port placement is shown. A primary port for camera insertion and held by the robotic arm is placed in the periumbilical area. Secondary $8 \mathrm{~mm}$ robotic port are placed at the lateral edge at the rectus muscle and an additional robotic $8 \mathrm{~mm}$ assistant port is placed in the left lower pelvis as shown. Two assistant ports (12 $\mathrm{mm}$ and $5 \mathrm{~mm}$ ) are placed in the right upper and lower pelvis respectively.

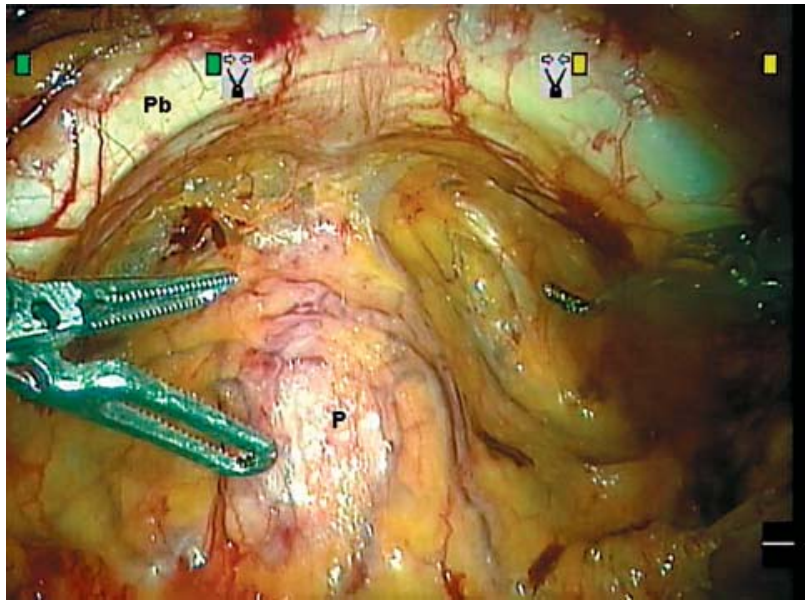

Figure 3 - Intraoperative image at the pelvis with the bladder dropped and prostate $(P)$ and pubic bone $(P b)$ exposed.

Dorsal vein complex (DVC) control: The fatty tissue covering the prostate is dissected and the endopelvic fascia and the anterior surface of the prostate is exposed. The superficial dorsal vein is precisely coagulated and divided with robotic bipolar cautery. The endopelvic fascia is then incised bilaterally, using no thermal energy with the robotic scissors. The DVC complex is then secured with 0 -vicryl on CT-1 needle, and divided at a later stage of surgery with the robotic J-hook (Figure-4). Urethral metal sound helps keeping the urethra away from the DVC during suturing. A second stitch is placed towards the prostatic base and used to control bladder neck vessels and aid in retraction of the prostate during the dissection of the bladder neck.

Division of the bladder neck: With the prostate retracted cephalad, the anterior bladder neck dissection is performed with the metal sound used to aid identify the bladder neck. An opening in the bladder neck is created with the robotic J-hook, and then with the third robotic arm retracting the prostate towards the abdominal wall, the bladder neck incision is prolonged horizontally. The assistant provides adequate visualization with suction, and appropriate traction during this step. The posterior aspect of the bladder neck is identified and dissected at the prostatovesical junction, starting from the midline and extended laterally to completely detach the bladder neck of the base of the prostate. In the presence of a large median lobe, 

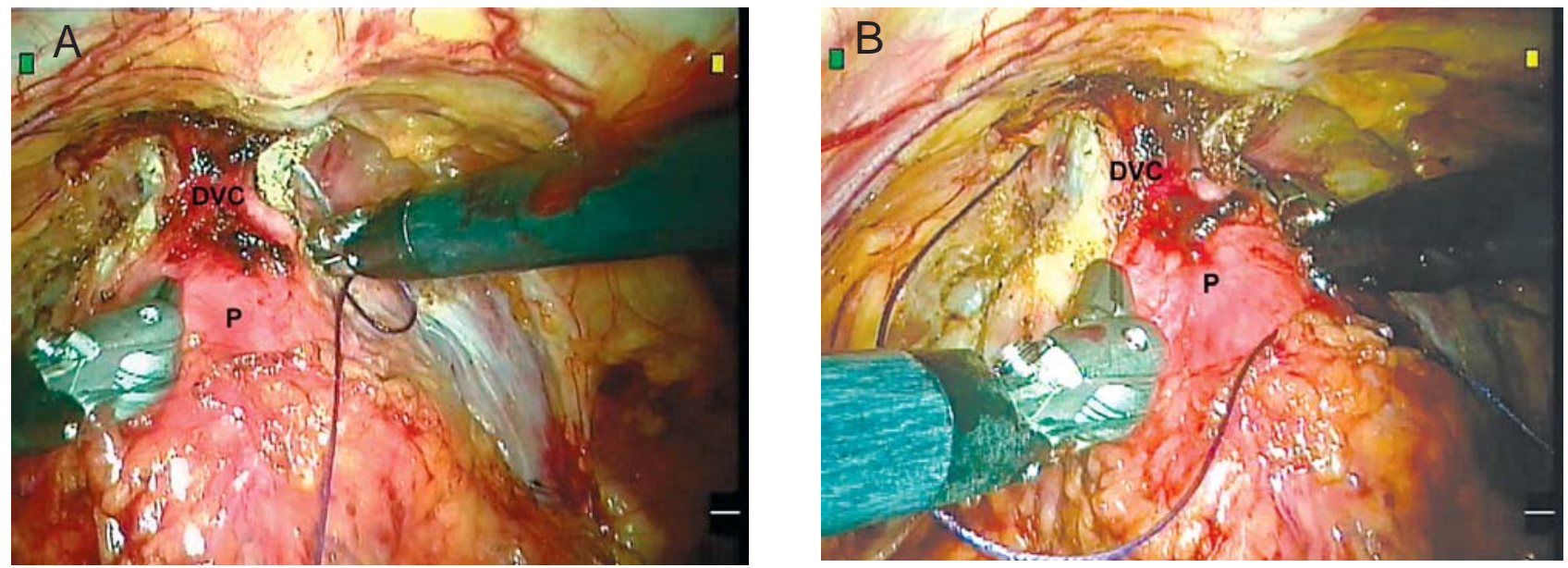

Figure 4-A) and B) - Intraoperative images illustrating the control of the dorsal vein complex. $P=$ prostate, $D V C=$ dorsal vein complex

ideally the ureteral meatus should be identified before initiating the posterior bladder neck incision (Figure$5)$.

Vas deferens and seminal vesicles dissection: After completing the dissection of the prostate base, both vas deferens are identified and cut, keeping a long stump of vas for retraction. The dissection continues by pulling the vas cephalad away from the rectum thus exposing the seminal vesicles that are located lateral to the vas on each side. Dissection is

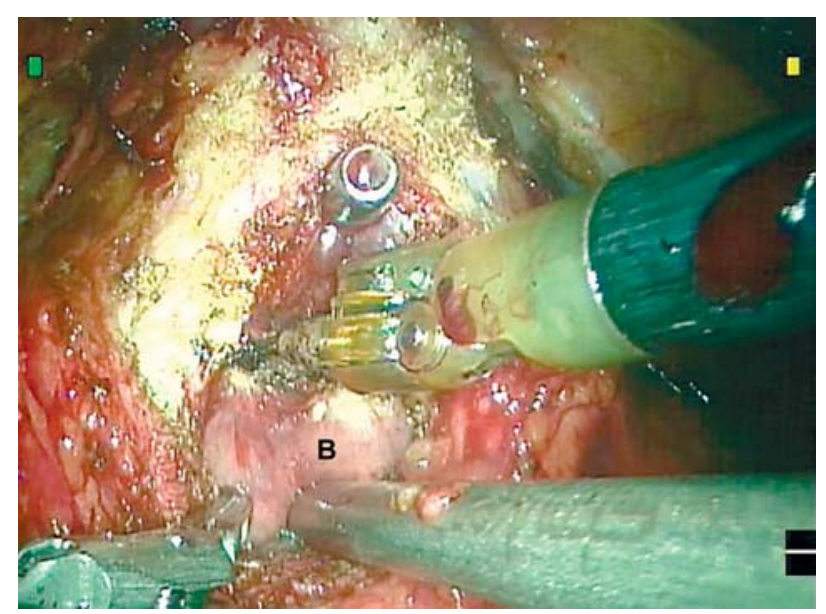

Figure 5 - Dissection of the bladder neck completed anteriorly while the posterior bladder neck is scored with electrocautery. $B=$ bladder neck. done mainly by blunt dissection with robotic scissors. When necessary $5 \mathrm{~mm}$ Hem-o-lock clips (Weck Closure Systems, Research Triangle Park, NC, USA), or harmonic scalpel are applied to control the seminal vesicular and vasal arteries. After a complete release of seminal vesicles and vas, the third robotic arm is used to pull up both seminal vesicles, while the assistant provides help with adequate suction, to expose the prostate lateral pedicle. The lateral prostatic pedicle is clipped or cut with harmonic scalpel (Figure-6).

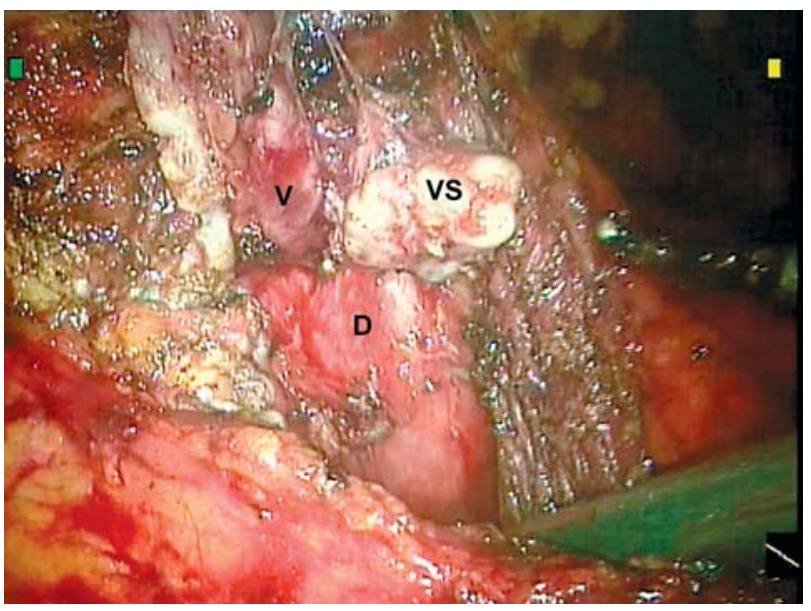

Figure 6-The vas $(V)$ is retracted cephalad exposing the ipsilateral seminal vesicle (SV) and Denonvilliers' fascia (D) after complete dissection of the bladder neck. 
Neurovascular bundle (NVB) dissection (nerve sparing): This step is performed under the view of a $0^{\circ}$ laparoscope lens, without employing any thermal energy to optimize the NVB preservation. The NVB is released from the posterolateral aspect of the prostate using a combination of sharp and blunt dissection, using robotic scissors and prograsper. Using robotic scissors, the superficial prostatic fascia is incised and the neurovascular bundle is peeled bluntly. This dissection is extended laterally and distally towards the prostate apex (Figure-7).

Prostate apex and urethral dissection: This step is crucial for postoperative continence and oncological control. The prostate is retracted cephalad out of the pelvis, and the prostate apex is bluntly dissected without compromise the NVB integrity with any thermal injury. The previously ligated DVC is cut using $\mathrm{J}$ hook cautery. The urethra is incised with robotic scissors anteriorly and then completely divided. A 10 mm Endocatch bag (US Surgical, Norwalk, CT, USA) is used for specimen store and retrieval at the end of the procedure.

Vesicourethral anastomosis: Two monocryl 20 sutures on a UR-6 needle are used to complete the vesicourethral anastomosis then the two sutures are tied to each other. The van Velthoven running suture technique is used, starting at 6 o' clock position in the

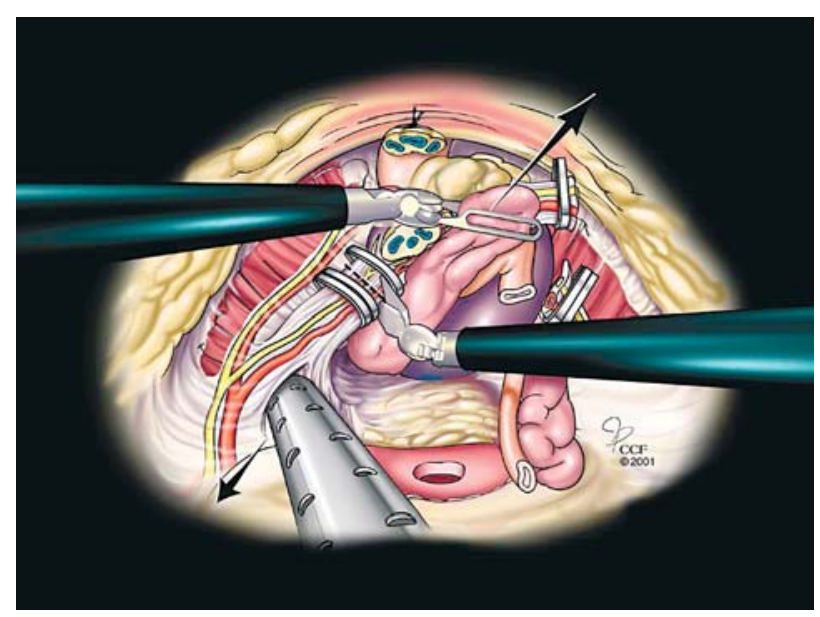

Figure 7 - Bladder neck has been dissected completely and vascular pedicle clipped and ready to be cut as show. Note that adequate traction of prostate (arrow) is important to bluntly peal the NVB. posterior bladder neck, progressing towards 12 o'clock, one in a clockwise and the other in an anti-clockwise fashion. The bladder is firstly brought close to the urethra when the anastomosis reaches 9 and 3 o'clock positions respectively, then a 20 Fr Foley catheter is inserted (Figures-8A and 8B).
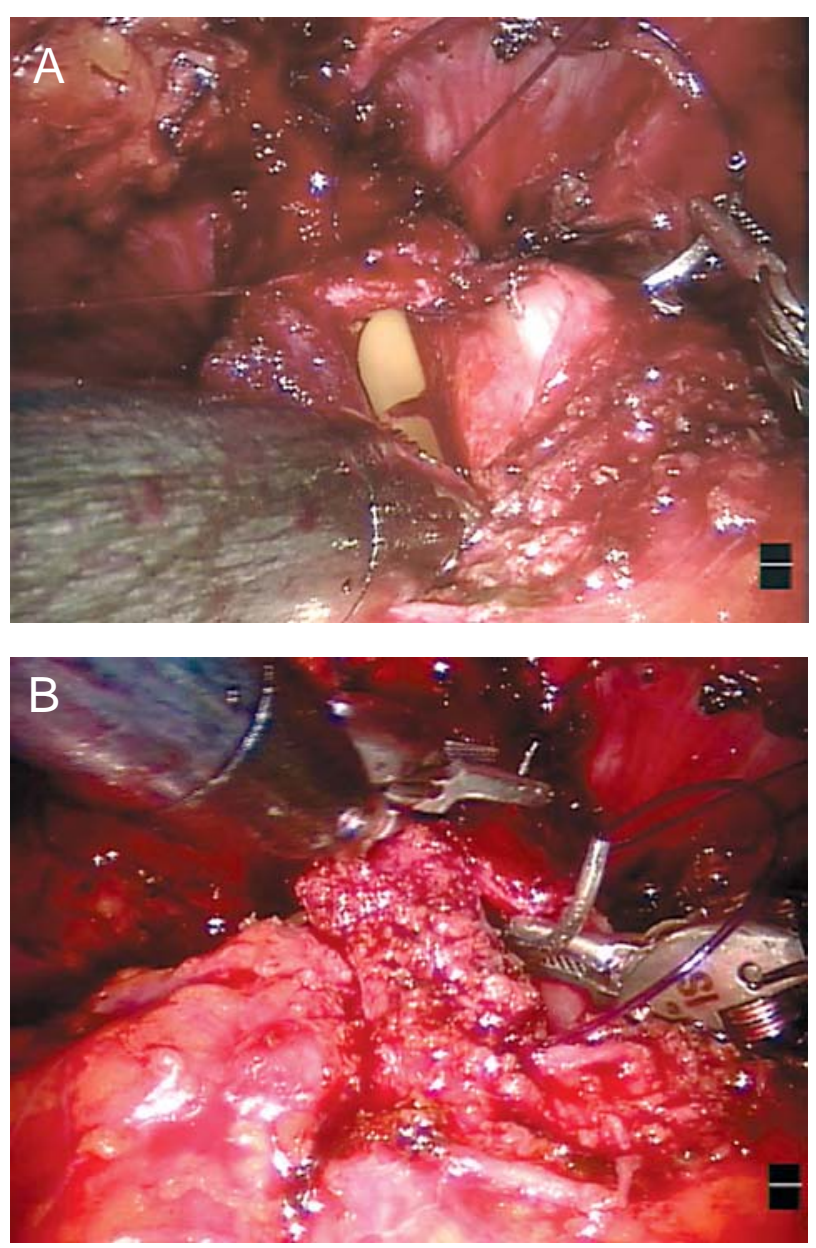

Figure 8-A) The vesicourethral anastomosis starts at $60^{\prime}$ 'clock position at the bladder neck. B) The urethral suture is taken as shown and the bladder neck is approximated to the urethra.

Ports closure: The retrieval of the laparoscopic bag with the intact specimen is made through the extension of the periumbilical $12 \mathrm{~mm}$ port site. A Jackson-Pratt drain is placed in the vicinity of the vesicourethral anastomosis through a lateral $5 \mathrm{~mm}$ port site. The Carter Thomason device is used to close the $12 \mathrm{~mm}$ port sites. 


\section{Extraperitoneal Approach}

Patient positioning: During this approach, the patient is positioned in supine position, with arms along the body in a similar fashion to the transperitoneal approach. The legs are separated in semi flexion (lithotomy position) with a 20F Foley catheter inserted into the bladder. The operating table is maintained in a slight Trendelenburg position for this approach.

Pneumoperitoneum: Using a $2 \mathrm{~cm}$ midline incision made $1 \mathrm{~cm}$ inferior to the umbilicus, the subcutaneous layer is divided deep to the anterior rectus fascia, which is incised transversally and separated in the linea alba. A limited extraperitoneal space is initially developed bluntly with index finger dissection along the posterior rectus sheath. This space is bordered caudally by the arcuate line of Douglas, posteriorly by the posterior rectus sheath, anteriorly by the posterior fibers of the rectus muscle. A balloon dissector (US surgical) is then introduced through the developed space and inflated gradually up to $400 \mathrm{cc}$ of air then deflated and removed.

Port placement: A special trocar (12 mm) with $30 \mathrm{cc}$ balloon mounted tip (US Surgical) is placed at the initial umbilical incision. Under direct vision, four additional trocar are placed: three $8 \mathrm{~mm}$ da Vinci trocars and an extra $12 \mathrm{~mm}$ regular port are positioned. The Retzius space is opened and the prevesical space is developed laterally. The anterior aspect of the bladder, the pubic arch and the external iliac vessels are visualized. The procedure is continued in a similar fashion as in the transperitoneal approach as describe above.

\section{RESULTS}

A prospective data collection at 250 RARP has been completed. The mean age of the patients was $60.5 \pm 6.9$ years, with BMI of $28.7 \pm 3.7$, mean preoperative PSA of $6.2 \pm 3.4 \mathrm{ng} / \mathrm{mL}$, and median Gleason score on the preoperative prostate biopsy of 6 (IQR 6,7). Overall, $9 \%$ of the patients had a previous abdominal surgery, with a median ASA score was 2 (IQR 2,3).

The mean operative time was $200 \pm 61 \mathrm{~min}$; of these, vesicourethral anastomosis took $24 \pm 11 \mathrm{~min}$ to perform. The estimated blood loss was $250 \mathrm{~mL}$ (IQR 150,350), with blood transfusion rate of $1.9 \%$, perioperative complication rate of $1.2 \%$, and overall positive margin rate of $12 \%$. Mean hospital stay was $1.8 \pm 1.1$ days since the admittance.

No significant difference was noted between the transperitoneal and the extraperitoneal approaches as regards blood loss, blood transfusion rate, operative time, intraoperative urine output, urethrovesical anastomosis time, positive margins, and complications.

\section{COMMENTS}

Since the introduction of the AESOP (Computer Motion, Goleta, California, USA) in 1994 and the da Vinci Surgical System in 1997, the introduction of surgical robots is growing in a fast pace. Over 150 hospitals in United States and Europe are currently performing robotic assisted surgery and this trend is growing (4).

The major drawback for robotic surgery is high cost, including the robotic system, disposable instruments, operating room facilities, training personnel, and maintenance. The multidisplinary use and marketing strategies are the main allies of the surgeon at the initiation of a robotic program, which depends on surgical volume and indirect referrals to the health center. Urology, gynecology, general surgery, and cardiothoracic surgery are currently using robotic assisted procedures regularly.

The minimally invasive approach offers less morbidity compared to open radical prostatectomy, and available data shows comparable results between the robotic assisted and pure laparoscopic techniques, with the former presenting a reduced learning curve for technically demanding operations, mainly due to three dimensional visualization and enhanced surgical dexterity (5-7). Improvement in available surgical robots will further enhance the wide spread use of robotics.

\section{CONFLICT OF INTEREST}

None declared. 


\section{REFERENCES}

1. Menon M, Tewari A, Peabody JO, Shrivastava A, Kaul S, Bhandari A, et al.: Vattikuti Institute prostatectomy, a technique of robotic radical prostatectomy for management of localized carcinoma of the prostate: experience of over 1100 cases. Urol Clin North Am. 2004; 31:701-17.

2. Abbou CC, Hoznek A, Salomon L, Olsson LE, Lobontiu A, Saint F, et al.: Laparoscopic radical prostatectomy with a remote controlled robot. J Urol. 2001; 165: 1964-6.

3. Pasticier G, Rietbergen JB, Guillonneau B, Fromont G, Menon M, Vallancien G: Robotically assisted laparoscopic radical prostatectomy: feasibility study in men. Eur Urol. 2001; 40: 70-4.

4. Steers WD, LeBeau S, Cardella J, Fulmer B: Establishing a robotics program. Urol Clin North Am. 2004; 31: 77380.
5. Bentas W, Wolfram M, Jones J, Brautigam R, Kramer W, Binder J: Robotic technology and the translation of open radical prostatectomy to laparoscopy: the early Frankfurt experience with robotic radical prostatectomy and one year follow-up. Eur Urol. 2003; 44: 175-81.

6. Ahlering TE, Skarecky D, Lee D, Clayman RV: Successful transfer of open surgical skills to a laparoscopic environment using a robotic interface: initial experience with laparoscopic radical prostatectomy. J Urol. 2003; 170: 1738-41.

7. Menon M, Tewari A, Baize B, Guillonneau B, Vallancien G: Prospective comparison of radical retropubic prostatectomy and robot-assisted anatomic prostatectomy: the Vattikuti Urology Institute experience. Urology. 2002; 60: 864-8.

\author{
Correspondence address: \\ Dr. Jihad H Kaouk \\ Section of Laparoscopic and Robotic Surgery \\ Glickman Urological Institute, Cleveland Clinic \\ 9500 Euclid Av, A100 \\ Cleveland, OH 44195 \\ Fax: + 1216445-2267 \\ E-mail:kaoukj@ccf.org
}

